Obuchowska Aleksandra, Standyło Arkadiusz, Ozga Alicja, Wójcik Justyna, Obuchowska Karolina, Skorupski Pawel. Neoadjuvant chemotherapy for IIIC stage ovarian cancer - case report. Journal of Education, Health and Sport. 2020;10(9):84-91. eISSN 23918306. DOI http://dx.doi.org/10.12775/JEHS.2020.10.09.009

https://apcz.umk.pl/czasopisma/index.php/JEHS/article/view/JEHS.2020.10.09.009

https://zenodo.org/record/4014352

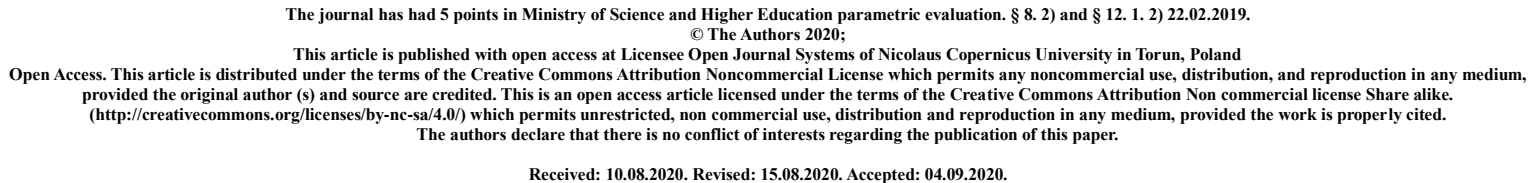

\title{
Neoadjuvant chemotherapy for IIIC stage ovarian cancer - case report
}

\author{
Aleksandra Obuchowska ${ }^{1 \mathrm{a}}$, Arkadiusz Standyło ${ }^{\text {1b }}$, Alicja Ozga ${ }^{1 \mathrm{c}}$, Justyna Wójcik ${ }^{1 \mathrm{~d}}$, \\ Karolina Obuchowska $^{1 \mathrm{e}}$, Pawel Skorupski ${ }^{2 \mathrm{f}}$
}

${ }^{1}$ Student Scientific Group at the $2{ }^{\text {nd }}$ Chair and Department of Gynecology, Medical University of Lublin, Lublin, Poland ${ }^{2} 2^{\text {nd }}$ Chair and Department of Gynecology, Medical University of Lublin, Lublin, Poland

a aobuchowska12@gmail.com; https://orcid.org/0000-0003-0464-2695

b a.standylo@gmail.com; https://orcid.org/0000-0002-5154-4759

c aozga1@gmail.com; https://orcid.org/0000-0003-1291-905X

d justynawojcik455@gmail.com; https://orcid.org/0000-0001-7163-6784

${ }^{\mathrm{e}}$ karolinaobuchowska99@ gmail.com; https://orcid.org/0000-0003-4519-8236

f pawskor@tlen.pl; https://orcid.org/0000-0002-1635-831X

\begin{abstract}
Introduction: Ovarian cancer accounts only for $4.7 \%$ of female malignancies in Poland but has the worst prognosis among gynaecological neoplasms. Due to non-specific and scant symptoms the disease is diagnosed in nearly $70 \%$ of cases in stages III and IV (FIGO). The primary goal of surgical treatment is complete cytoreduction with no residual disease left after surgery involving hysterectomy, bilateral adnexectomy, omentectomy and removal of all metastatic foci from the peritoneum and abdominal organs. If it is not possible to achieve the complete cytoreduction, patients may be qualified for neoadjuvant chemotherapy (NACT) and surgery after achieving complete or partial remission.
\end{abstract}


Purpose: Description of NACT protocol used for the treatment of stage IIIC ovarian cancer. Comparison of treatment results of advanced ovarian cancer using NACT and primary debulking surgery (PDS).

Case report: A 58-year-old female patient was hospitalized with the diagnosis of the pelvic mass. Her predominant signs were abdominal pain on the right flank and flatulence. Laboratory tests and imaging studies indicated a high risk of metastatic ovarian cancer. Initial diagnosis was confirmed on the laparoscopy. Ovarian cancer matched stage IIIC with Fagotti score of 8 points. The inspection of the abdominal organs revealed tumors in both adnexa, miliary spread on the parietal peritoneum, the surface of the small intestine and the diaphragm and the thick infiltration of greater omentum. Multiple specimens for the pathology were collected and left adnexectomy was done. Due to the inability to achieve complete cytoreduction, the patient was qualified to NACT. Following 6 cycles of standard protocol paclitaxel and carboplatin chemotherapy, laparotomy with hysterectomy, bilateral adnexectomy and omentecomy was done. Residual disease was found only within greater omentum but complete cytoreduction was achieved. The patient was discharged in good condition.

Summary: Clinical data indicates that patients with advanced ovarian cancer treated with NACT and surgery have similar prognosis to those treated by primary cytoreductive surgery. The advantages of NACT are reduced perioperative morbidity and mortality, lower complication rate and shorter hospital stay. In the described patient, the implementation of the NACT protocol has resulted in complete surgical cytoreduction which is the main factor permitting prolongation of life expectancy in woman with advanced ovarian cancer.

Key words: ovarian cancer; neoadjuvant chemotherapy; cytoreductive surgery; postoperative complications

\section{Introduction:}

Ovarian cancer accounts only for $4.7 \%$ of female malignancies in Poland but has the worst prognosis among gynaecological neoplasms. Morbidity is age-related, $80 \%$ of ovarian cancer cases occur after the age of fifty with over $50 \%$ being diagnosed between 50 and 69 years of age. The median age at diagnosis is 63 years (range 55-64) and the median age of death is 71 years (range 65-74) [1]. Due to non-specific and scantily expressed symptoms $75 \%$ of patients have metastatic disease at the time of diagnosis [2]. Five-year survival rate for patients with stage I is 70-80\%, whereas in patients with stage IV disease only $15 \%$ [3]. The primary goal of the ovarian cancer surgical treatment is complete cytoreduction with no residual disease left after intervention involving hysterectomy, bilateral adnexectomy, omentectomy and removal of all metastatic foci from the peritoneum and abdominal organs. Imaging studies, serum levels of the tumor markers and laparoscopy are the tools employed in estimation of the likelihood of completeness of the cytoreductive procedure [4]. When the extensive neoplastic spread precludes chances of favourable surgical outcome patients can be treated with neoadjuvant chemotherapy (NACT) following by surgery after achievement of complete or partial remission $[4,5]$. 


\section{Purpose:}

Description of NACT protocol used for the treatment of stage IIIC ovarian cancer. Comparison of treatment results of advanced ovarian cancer using NACT and primary debulking surgery (PDS).

\section{Case description:}

A 58-year old patient visited primary care physician due to severe abdominal pain in the right flank radiating to the spine and flatulence. Serum levels of cancer markers were elevated - CA-125 - $754 \mathrm{U} / \mathrm{ml}, \mathrm{HE} 4-758 \mathrm{pmol} / \mathrm{l}$ and were highly suspicious of the ovarian malignancy ( ROMA - 97.5\%) [6]. Serum level of CA 15-3 was $48 \mathrm{U} / \mathrm{ml}$ which also exceeded the upper limit, CEA level that was within the normal range $-0.8 \mathrm{ng} / \mathrm{ml}$. The patient was in a good condition and was admitted to the Department of Gynaecology due to suspected ovarian cancer. Her medical history was uneventful. Patient was not cachectic her BMI was 24 $\mathrm{kg} / \mathrm{m}^{2}$. Menarche appeared at the age of 14 and menopause at the age of 50. Patient gave once vaginal birth. Last PAP smear was negative but most recent one was done 8 years before admission. Transvaginal ultrasound revealed a cystic-solid lesion with dimensions of $54 \times 43$ $\mathrm{mm}$ in the right adnexa and similarly structured lesion sized $87 \times 72 \mathrm{~mm}$ in the left adnexa. Sonography of the upper abdomen showed possible massive carcinomatous involvement of greater omentum. Diagnosis of metastatic ovarian neoplasm was confirmed on computed tomography (CT) [Figure 1,2].

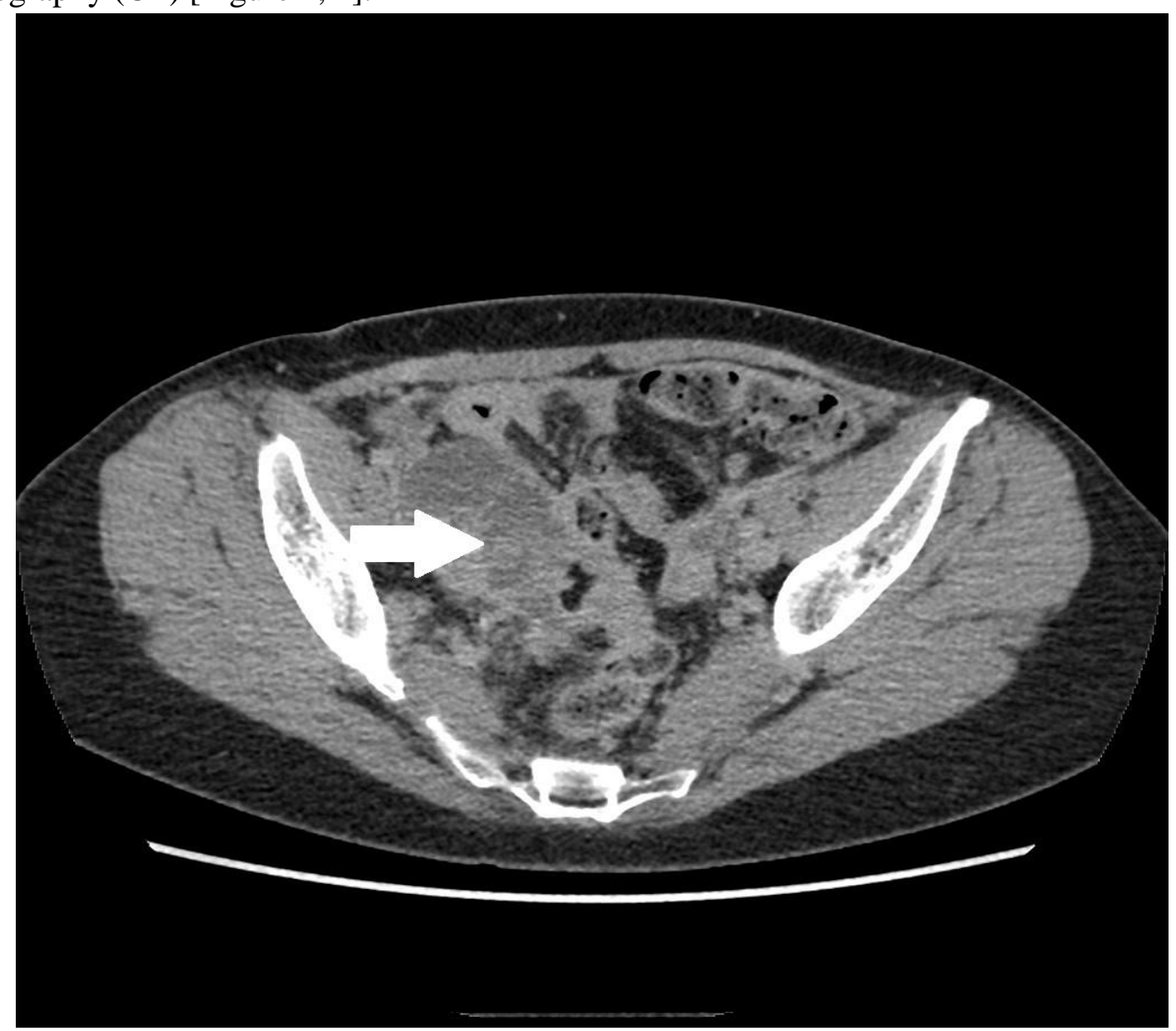

Figure 1. Cystic-solid tumor of the right ovary. 


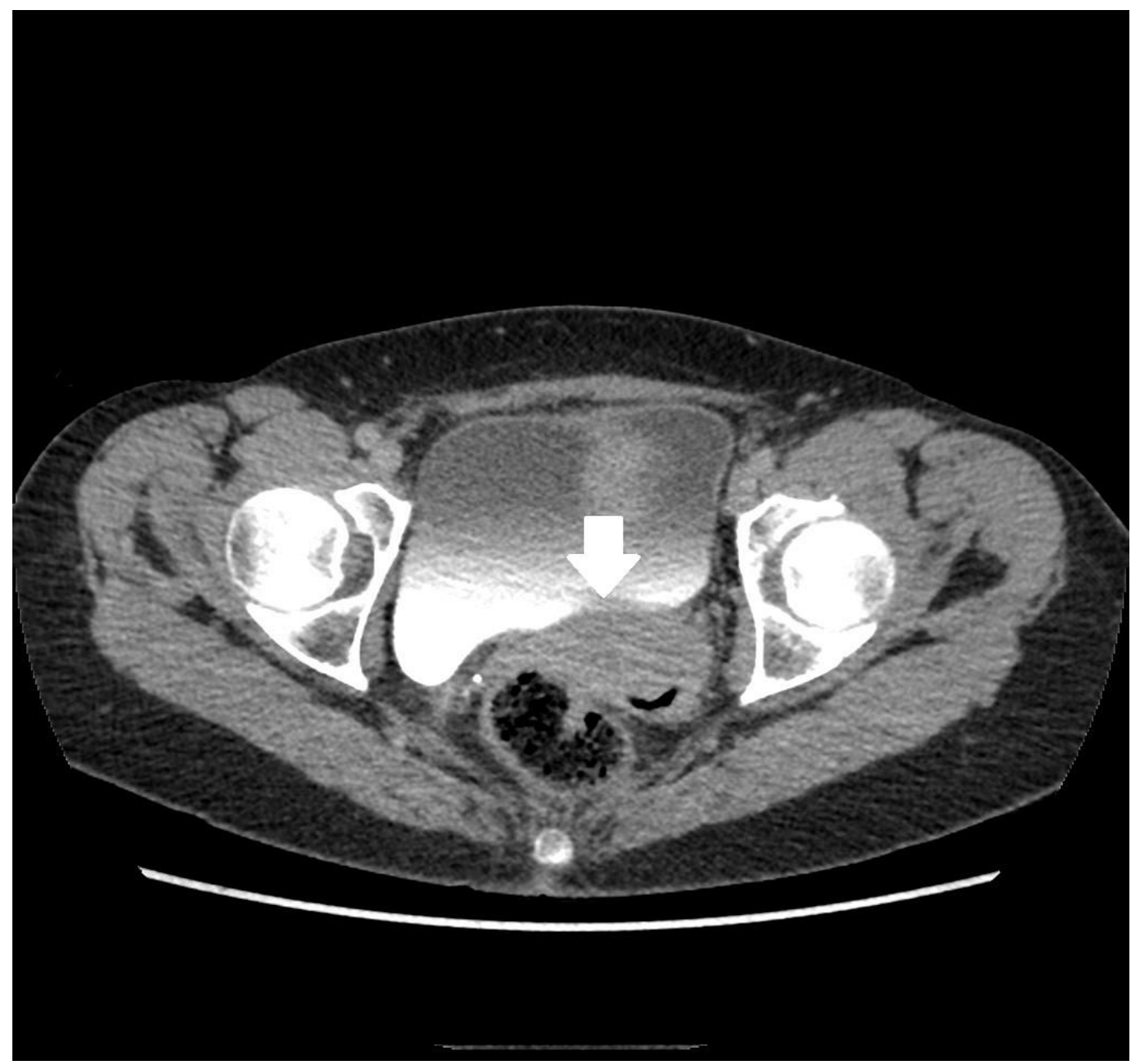

Figure 2. Blurred boundary between the bladder and uterus indicates possible neoplastic involvement of the urinary bladder.

The CT scan showed pelvic masses located in the both adnexa and peritoneal metastases in middle and upper abdomen with a diameter exceeding $2 \mathrm{~cm}$. Since the operability of a such advanced disease is not guaranteed patient was qualified for diagnostic laparoscopy. Intraabdominal inspection revealed about $200 \mathrm{ml}$ of ascitic fluid, extensive miliary carcinomatosis on the parietal peritoneum and small intestine and on the visible on the surface of the diaphragm. The surface of the liver was clear, the stomach looks normal. Greater omentum was extensively infiltrated (omental cake) and fixed to the parietal peritoneum. Sigmoid colon firmly attached to the lateral abdominal wall. Uterus and adnexa initially invisible due to neoplasmatic infiltration and massive adhesions. Their release have partially opened pouch of Retzius with immobile uterus attached firmly to the bladder and rectum. Peritoneum covering urinary bladder was also infiltrated by cancer. Pelvis was completely obliterated by the ovarian tumors forming firm adhesions with rectum and sigmoid colon. Left-sided adnexectomy was done, numerous tissue samples from metastatic foci were taken and sent for pathological examination. 


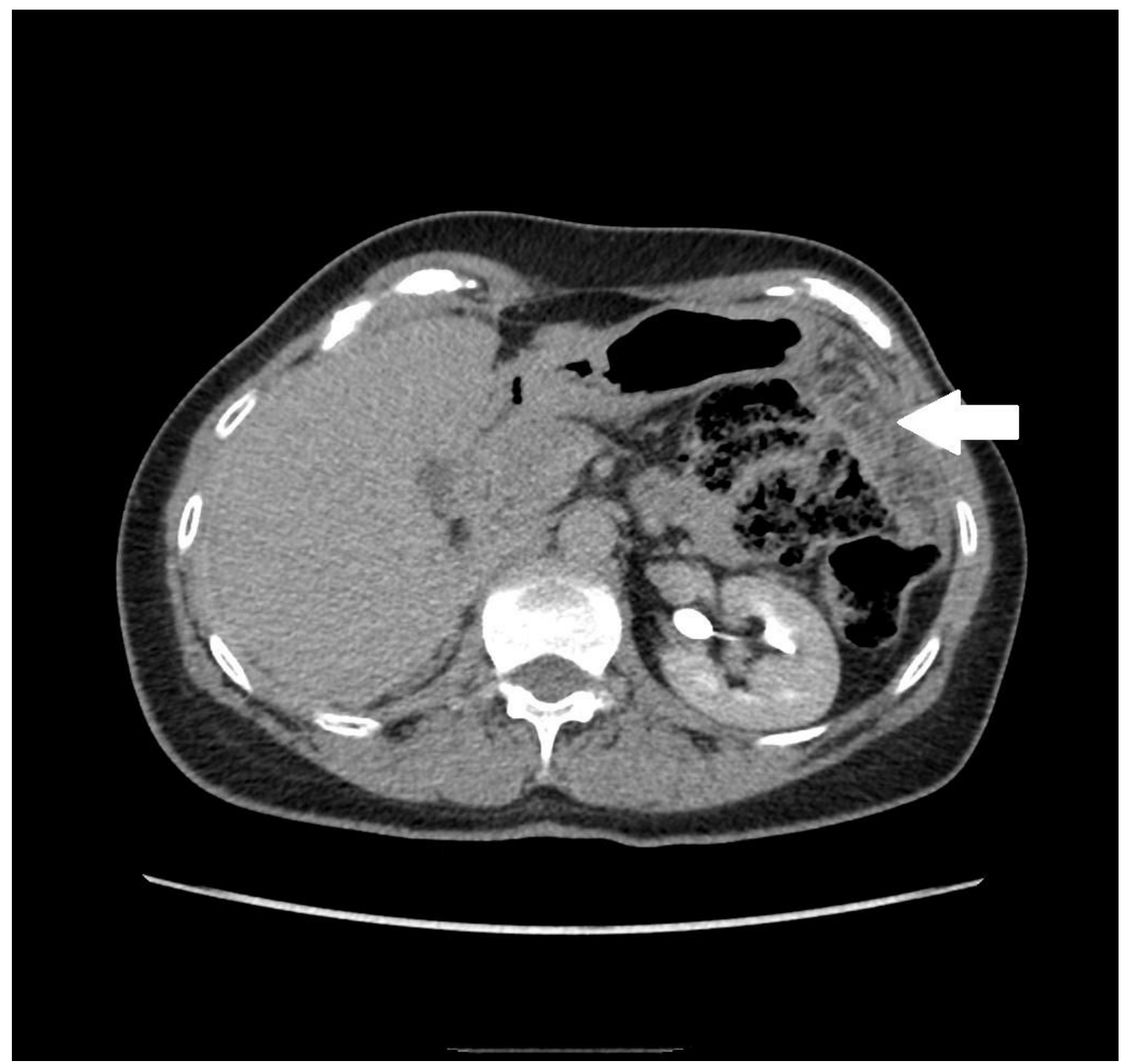

Figure 3. CT scan showing infiltration of the greater omentum "omental cake".

Staging according to FIGO classification was assessed as III C whereas Fagotti Score was estimated at 8 points $[4,5]$. On day 3 patient was discharged in good condition. Pathology report confirmed clinical diagnosis of ovarian cancer. The microscopic image corresponds to the diagnosis of high-grade serous ovarian cancer. Grade 3 tumor formed papillary structures and its structure was partially solid with the presence of necrosis foci, oviduct with the presence of serous cancer infiltrate. Due to the inability to obtain complete cytoreduction patient was scheduled for NACT. Following 6 cycles of paclitaxel/carboplatin chemotherapy the complete remission as based on clinical examination, CT scan and serum levels of cancer markers was achieved [7]. Patient was readmitted for surgical treatment in a good general condition and with proper level of nutrition. Standard surgical protocol with the extended vertical median incision, hysterectomy, adnexectomy, omentectomy was performed. Upon direct visualisation there was no residual disease in the abdominal organs except the greater omentum where nodular lesions with a diameter of 5 to $15 \mathrm{~mm}$ were seen. Parietal peritoneum, uterus, right adnexa, small and large intestine, and other abdominal organs were clear of the disease, thin adhesions partially obliterated recto-uterine pouch. 
Complete cytoreduction (R0) was obtained. The surgery and the postoperative period went without complications and the patient was discharged in good condition.

\section{Rationale for the study}

Early ovarian cancer is usually clinically silent thus $60-70 \%$ of cases are diagnosed in advanced stages that basically preclude the patient from being completely cured [2]. Early symptoms if exist are non-specific and include dyspepsia which can occur even a year before the diagnosis $[5,8]$. Universal population screening is not recommended, except in woman with $B R C A$ 1, 2 genes mutations. Ovarian cancer is characterised by the insidious onset and aggressive clinical course and therefore requires interdisciplinary approach and the precise collaboration between the gynaecologist and oncologist during the diagnostics and treatment. The mainstay of the treatment is primary cytoreductive surgery and subsequent chemotherapy. The most important factor influencing 5-year survival rate is quality of the surgery. So the main goal of the ovarian cancer surgery is removal of all macroscopic disease. If not possible, optimal cytoreduction could be attempted which resulted in leaving cancer foci smaller than $1 \mathrm{~cm}$ [9]. Two studies conducted by GOG (Gynecologic Oncology Group) showed that diameter of residual tumor influences the survival rate of patients. Four years overall survival is reported in $60 \%$ of patients with only microscopic residual disease, $40 \%$ of patients with less than $2 \mathrm{~cm}$ residual disease and less than $20 \%$ of patients with unexcised tumor exceeding $2 \mathrm{~cm}$. Proper selection of the patients with no chances of achieving complete or at least optimal cytoreduction because of the advancement of the disease is a basic step in preoperative work-up [10,11]. Nowadays there is no imaging technique $100 \%$ sensitive and accurate in diagnosing of abdominal burden of the disease. When issues concerning operability arises it is indicated to perform diagnostic laparoscopy prior to definitive surgical treatment. During the procedure systematic evaluation of abdominal organs is made as described by Fagotti group [4]. When the estimated score is less than or equal to 8, patient is qualified for primary debulking surgery (PDS). The outcome of more than 8 points indicate that PDS is not feasible. Instead multiple biopsies are taken and patient becomes candidate to neoadjuvant chemotherapy and surgery (NACT) after obtaining complete or partial remission of the disease [4,5]. Non-optimal treatments significantly shorten the time to progression and negatively influence overall survival [12]. NACT with standard protocol of chemotherapy with paclitaxel and carbo- or cisplatin with added bevacizumab (if the patient meets the eligibility criteria for bevacizumab therapy) enabled symptomatic and biochemical improvement and reduction of size or disappearance of the tumor. Following favorable clinical response the cytoreductive surgical treatment (interval debulking surgery, IDS) should be scheduled after 3-4 courses of chemotherapy. This strategy seemed to increase optimum debulking rates and reduce surgery related complications. In women with stage III or IV ovarian cancer, survival with primary chemotherapy is non-inferior to primary surgery and chemotherapy protocol [2]. In the case of ovarian cancer stages III and IV, the residual disease below $1 \mathrm{~cm}$ is possible to achieve in $41 \%$ of patients in the case of PDS and in $73 \%$ in the case of IDS $(\mathrm{p}=0.0001)$ [2]. CHORUS study proved that in stage III complete cytoreduction could be achieved during primary surgery in $16 \%$ of cases, whereas NACT protocol increase this value to $43 \%$ [2]. 
Incomplete cytoreduction (residual disease $>1 \mathrm{~cm}$ ) was achieved in $59 \%$ of patients in primary surgery and in $24 \%$ of treated following primary chemotherapy [2]. The residual disease of $0 \mathrm{~cm}$ in stage IV was obtained in $18 \%$ of patients on primary surgery and in $29 \%$ cases of primary chemotherapy [2]. The residual disease of less than $1 \mathrm{~cm}$ was obtained in $24 \%$ in primary surgery and in $37 \%$ during primary chemotherapy [2]. Incomplete cytoreduction was noted in 58\% of patients in primary surgery group and in $35 \%$ of woman after primary chemotherapy [2]. Progression-free survival was 12 months for NACT and 10.7 months for PDS combined in stages III and IV ( $\mathrm{p}=0.2923)$ [2]. The 3-year survival rate was $32 \%$ in the primary surgery group versus $34 \%$ in primary chemotherapy group [2]. Median overall survival was subsequently 22.6 and 24.1 months (HR 0.87 in favour of women assigned to primary chemotherapy) [2]. In the case of PDS, in which obtaining the cytoreduction of $<1 \mathrm{~cm}$ was not possible ( $58.4 \%$ of cases), the overall survival (OS) has been limited to 34 months [13]. In IDS, where in most of the cases (80.6\%) successful surgery resulted in residual disease $\leq 1 \mathrm{~cm}$, OS totals 50 months [13]. Obviously, if there is a possibility of achieving at least optimal cytoreduction in the case of PDS, OS is significantly longer and totals from 50 up to 66 months for residual disease of $\leq 1 \mathrm{~cm}$ and 65 to 110 months for residual disease of $0 \mathrm{~cm}$ [13]. The number of surgical procedures performed during PDS and IDS differs significantly. More large intestine resections are conducted during PDS $(46.2 \%)$ than IDS $(27.5 \%)(\mathrm{p}=0.028)$ [9]. The number of diaphragm resections is also higher in PDS (13.9\%) than in IDS (7.5\%) $(\mathrm{p}=0.33)$ [9]. The number of surgical and postsurgical complications is greater in the case of PDS, including intestinal complications, thromboembolism, organ failure, postoperative bleeding, pulmonary complications. These complications increase the risk of postoperative death. Causes of death after debulking surgery are both surgical complications (hemorrhage, sepsis, bowel perforation) - 29\%, systemic complications - 42\% (pulmonary failure - 4\%, cardiac complications - $13 \%$, thrombo-embolism $-25 \%$ ) and others - 29\% (bowel obstruction, renal failure, stroke, progressive disease [9]. The only complication that is more common following IDS then PDS are infections most probably caused by chemotherapy induced leucopenia. The postsurgical mortality (POM) is generally defined as death from any cause within 30 days of operation. POM in the primary chemotherapy group was less than $1 \%$, whereas $3.7 \%$ (range $2.5-4.8 \%$ ) of patients after primary cytoreductive surgery [14]. Risk of POM is elevated in elderly women and after extensive procedures. In the primary chemotherapy group, $93 \%$ of patients were discharged within 14 days from operation, compared with only $80 \%$ of them in the primary surgery group [14].

\section{Summary}

The clinical efficacy of NACT protocol in terms of overall survival in patients with advanced ovarian cancer is comparable to this parameter in the group subjected to primary cytoreductive surgery. Main benefits of NACT is reduction of peri- and postoperative morbidity and mortality, limiting the rate of surgical and postsurgical complications and shortening duration of hospital stay. Quality of life in oncological patients is a significant issue related directly to the treatment modality. 
In the described patient it was possible to achieve a complete surgical cytoreduction without complications related to the extensive surgery. Therefore we can conclude that NACT protocol benefited patient's quality of life while preserving her life expectancy.

\section{References:}

1. Wojciechowska U, Didkowska J. Zachorowania i zgony na nowotwory złośliwe w Polsce. [Incidence and deaths of malignant cancer in Poland]. Krajowy Rejestr Nowotworów, Narodowy Instytut Onkologii im. Marii Skłodowskiej-Curie - Państwowy Instytut Badawczy. Available at: http://onkologia.org.pl/raporty/, access of the day: 10/03/2020.

2. Kehoe S, Hook J, Nankivell M, et al. Primary chemotherapy versus primary surgery for newly diagnosed advanced ovarian cancer (CHORUS): an open-label, randomised, controlled, non-inferiority trial. Lancet 2015; 386(9990):249-57.

3. Lalwani N, Prasad S, Vikram R, Shanbhogue A, Huettner P, et al. Histologic, molecular, and cytogenetic features of ovarian cancers: implications for diagnosis and treatment. Radiographics 2011; 31: 625646.

4. Fagotti A, Ferrandina G, Fanfani F, Garganese G, Vizzielli G, et al. Prospective validation of a laparoscopic predictive model for optimal cytoreduction in advanced ovarian carcinoma. Am J Obstet Gynecol 2008; 199:642.e1-642.e6.

5. Basta A, Bidziński M, Bieńkiewicz A, Blecharz P, Bodnar L, et al. Recommendations of the Polish Gynecological Oncology Society for the diagnosis and treatment of ovarian cancer. Gynecol Oncol 2017; 15(1), $5-23$.

6. Geomini P, Kruitwagen R, Bremer GL et al. The accuracy of risk scores in predicting ovarian malignancy: a systematic review. Obstet Gynecol 2009; 113: 384-394.

7. Konstantinopoulos P, Ceccaldi R, Shapiro G, D'Andrea A. Homologous recombination deficiency: Exploiting the fundamental vulnerability of ovarian cancer. Cancer Discov 2015; 5(11): 1137-1154.

8. Kujawa K, Lisowska K. Ovarian cancer - from biology to clinic. Postepy Hig Med Dosw 2015; 69:1275-90.

9. Sehouli J, Savvatis K, Braicu E, Schmidt S, Lichtenegger W, et al. Primary Versus Interval Debulking Surgery in Advanced Ovarian Cancer: Results From a Systematic Single-Center Analysis. Int J Gynecol Cancer 2010; 20(8):1331-40.

10. Hoskins WJ, McGuire WP, Brady MF, et al. The effect of diameter of largest residual disease on survival after primary cytoreductive surgery in patients with suboptimal residual epithelial ovarian carcinoma. Am J Obstet Gynecol 1994; 170(4):974-980. doi:10.1016/s0002-9378(94)70090-7.

11. Hoskins WJ, Bundy BN, Thigpen JT, Omura GA. The Influence of Cytoreductive Surgery on Recurrence-Free Interval and Survival in Small-Volume Stage III Epithelial Ovarian Cancer: A Gynecologic Oncology Group Study. Gynecol Oncol 1992; 47: 159-166.

12. Elattar A, Bryant A, Winter-Roach B, et al. Optimal primary surgical treatment for advanced epithelial ovarian cancer. Cochrane Database Syst Rev 2011; (8):CD007565.

13. Bristow RE, Tomacruz RS, Armstrong DK, Trimble EL, Montz FJ. Survival effect of maximal cytoreductive surgery for advanced ovarian carcinoma during the platinum era: a meta-analysis. J Clin Oncol 2002; 20(5):1248-1259. doi:10.1200/JCO.2002.20.5.1248.

14. Gerestein C, Damhuis R, Burgerc C, Kooi G. Postoperative mortality after primary cytoreductive surgery for advanced stage epithelial ovarian cancer: A systematic review. Gynaecologic Oncology 2009; 114(3):523-7. 